\title{
Broadband Tunable Terahertz Beam Deflector Based on Liquid Crystals and Graphene
}

\author{
Yanchun Shen ${ }^{1,2}$, Jinlan Wang ${ }^{1}$, Qiaolian Wang ${ }^{1}$, Ximing Qiao ${ }^{1}$, Yuye Wang ${ }^{2, *}$ and Degang $\mathrm{Xu}^{2, *}$ \\ 1 Information Engineering Institute, Guangzhou Railway Polytechnic, Guangzhou 510432, China; \\ shenyanchun@gtxy.edu.cn (Y.S.); wangjinlan@gtxy.edu.cn (J.W.); wangqiaolian@gtxy.edu.cn (Q.W.); \\ qiaoximing@gtxy.edu.cn (X.Q.) \\ 2 Institute of Laser and Optoelectronics, School of Precision Instruments and Optoelectronics Engineering \\ Tianjin University, Tianjin 300072, China \\ * Correspondence: yuyewang@tju.edu.cn (Y.W.); xudegang@tju.edu.cn (D.X.)
}

Citation: Shen, Y.; Wang, J.; Wang,

Q.; Qiao, X.; Wang, Y.; Xu, D.

Broadband Tunable Terahertz Beam Deflector Based on Liquid Crystals and Graphene. Crystals 2021, 11, 1141. https://doi.org/10.3390/cryst11091141

\section{Academic Editors:}

Vladimir Chigrinov, Qi Guo,

Jiatong Sun and Ying Ma

Received: 7 August 2021

Accepted: 14 September 2021

Published: 18 September 2021

Publisher's Note: MDPI stays neutral with regard to jurisdictional claims in published maps and institutional affiliations.

Copyright: () 2021 by the authors. Licensee MDPI, Basel, Switzerland. This article is an open access article distributed under the terms and conditions of the Creative Commons Attribution (CC BY) license (https:// creativecommons.org/licenses/by/ $4.0 /)$.

\begin{abstract}
Terahertz (THz) technology has unique applications in, for example, wireless communication, biochemical characterization, and security inspection. However, high-efficiency, low-cost, and actively tunable $\mathrm{THz}$ modulators are still scarce. We propose a broadband tunable $\mathrm{THz}$ beam deflector based on liquid crystals (LCs). By a periodic gradual distribution of the orientation of the LC in one direction, a frequency-independent geometric phase modulation is obtained. The LC device with this specific orientation distribution was obtained through ultraviolet polarization exposure. We have verified the broadband beam deflection in both the simulation and experiment. The device can achieve a good spin-coupled beam deflection effect in the 0.8-1.2 Thz band, and the average polarization conversion efficiency exceeds $70 \%$. Moreover, because the electro-optical responsivity of LCs is excellent, graphene transparent electrode layers introduced on the upper and lower substrates enable the deflection modulation to be switched and dynamic tuning to be achieved.
\end{abstract}

Keywords: metasurface; liquid crystal; graphene; broadband tunable; beam deflector; terahertz

\section{Introduction}

The terahertz (THz) band is between 0.1 and $10 \mathrm{THz}$ (wavelength: 30-3000 $\mu \mathrm{m}$ ). Because the electromagnetic characteristics are unique, $\mathrm{THz}$ technology has broad applications in many fields, such as security inspection, biomedicine, and high-speed wireless communications [1-6]. Compared with THz sources and detectors, high-performance modulators used to transmit and control $\mathrm{THz}$ signals are still in the preliminary stage of development. Usually either a prism or a tilted phase plate, the beam deflector is one of the basic components for modulating $\mathrm{THz}$ signals and has wide applications in $\mathrm{THz}$ communication systems. The electrically tunable beam deflector using LC steers the incident beam at a desired angle by changing the refractive index of LC to form a phase profile [7-9]. The underlying principle of a traditional refractive optical element is the accumulation of the optical path difference in the propagation direction [10]. This device has a large volume and realizing a miniaturized and integrable version is difficult. Recently, researchers have developed THz beam deflectors based on metasurfaces [11,12], which are composed of sub-wavelength resonator arrays. The wavefront control is accomplished by introducing an abrupt phase change on a twodimensional planar surface, thereby attaining a thin and light structure. The shortcomings of such deflectors are a narrow modulation band and the lack of tunability. A tunable $\mathrm{THz}$ beam deflector is desirable to realize dynamic $\mathrm{THz}$ beam deflection that has high efficiency and a wide operating band to meet the needs of practical applications.

Dynamic modulation of terahertz beams can be achieved with many materials, such as semiconductors, superconductors, Phase Change Materials (PCM), Micro Electro Mechanical Systems (MEMS). Semiconductor materials, such as silicon and gallium arsenide (GaAs), can change their carrier concentrations by applying electric fields or optical pumping. The 
dielectric constant of semiconductor materials is determined by the carrier concentration, so the semiconductor materials are introduced into the super surface, thus realizing the dynamic control of electromagnetic characteristics. In 2013, Shrekenhamer et al. proposed an all-electronic multi-color spatial light modulator with super-pixels composed of arrays of $\mathrm{THz}$ metasurface absorbers. By combining a GaAs with the metasurface, an average modulation depth of $62 \%$ is achieved [13]. In 2010, Chen et al. used high-temperature superconducting films to replace metallic materials for the preparation of Split Ring Resonator (SRR) arrays. When the temperature is changed, the conductivity of the superconductor changes significantly, which can achieve an intensity tuning and a shift of resonant frequency. The operating frequency is $0.6 \mathrm{THz}$ and the operating bandwidth is about $60 \mathrm{GHz}$ [14]. This device is costly, as it requires a low-temperature action environment with superconducting materials as the modulating medium. In 2016, He et al. proposed a metallic metasurface on a $\mathrm{VO}_{2}$ film for $\mathrm{THz}$ wave focusing at $0.75 \mathrm{THz}$, and as the temperature increases from $293 \mathrm{~K}$ to $353 \mathrm{~K}, \mathrm{VO}_{2}$ changed from a dielectric to a metallic state because of a phase transition, which allowed the device to achieve switchable focusing modulation [15]. MEMS-based modulation uses external fields (e.g., temperature, electrostatic or piezoelectric drive) to modulate the bending of the cantilever of the structural unit to achieve a change in resonant characteristics. In 2011, Fu et al. achieved a frequency modulation bandwidth of $31 \%$ of the incident TE wave by adjusting the distance between resonant units and successively adjusting the resonant coupling strength by MEMS. The operating frequency point is located near $1.2 \mathrm{THz}$, and the whole structure is more compact. However, the preparation cost of the MEMS structure is higher, and the modulation efficiency of the single-layer metal resonance structure is lower [16].

In short, $\mathrm{THz}$ liquid crystal devices have some advantages in terms of modulation efficiency, bandwidth, and cost. However, the modulation rate of the liquid crystal is slow, about several millisecond, while the modulation rate of semiconductors and phase change materials can reach microseconds or even nanoseconds.

The polarization conversion efficiency of a single-layer metallic metasurface is less than $25 \%$ [17]. Subsequent improvements to metasurface efficiency include the use of multilayer metal metasurfaces and dielectric metasurfaces, and the efficiency is mostly below $80 \%$ [18-22]. While the liquid crystal is non-resonant, and the theoretical polarization conversion efficiency can reach more than $95 \%$ after optimizing the liquid crystal layer thickness and using low loss liquid crystal materials. This method has the advantage of relatively simple processing compared with the metasurface.

Liquid crystals (LCs) have mature applications in the field of flat-panel displays because of the electro-optical tunability and broadband birefringence $[23,24]$. In addition, the advantages of LC applications in non-display fields are gradually becoming apparent. For example, in the visible band, LC spatial light modulators and LC-on-silicon and other devices have been used for beam shaping, switching, and filtering $[25,26]$. In the early days, $\mathrm{THz} \mathrm{LC}$ devices mainly use magnetic fields or temperature control to change the refractive index to modulate the phase and polarization, and achieve functions such as filtering and phase shifting $[27,28]$. In recent years, with the development of $\mathrm{THz}$ transparent electrode materials and alignment technology for large cell thickness, electrically controlled LC waveplates and geometric phase components have been reported [29-34]. LC geometric phase elements use the spatial orientation distribution to obtain phase modulation. Compared with metasurface devices based on the geometric phase, LCs do not generate electromagnetic resonances, and its polarization conversion efficiency only depends on the phase retardation. Therefore, by selecting a reasonable cell thickness, the modulation efficiency is much higher than that of the metasurface. In addition, under an external electric field, the orientation direction of the LC is deflected, thereby changing the phase retardation accordingly [35]. Different voltages can be used to achieve high-efficiency modulation at different frequencies and to switch device functions. If the LC geometric phase device is applied to the $\mathrm{THz}$ beam deflection, a broadband tunable function can be realized. 
Here, we propose an LC-based broadband tunable $\mathrm{THz}$ beam deflector. The inner sides of the upper and lower quartz substrates are covered with graphene and a photoalignment layer, respectively. An LC layer with a thickness of $500 \mu \mathrm{m}$ lies in the middle. The orientation of the LC is realized with photo-alignment technology, which achieves a periodic gradual grating phase in a specific direction to realize the deflection of the $\mathrm{THz}$ beam. We have verified this deflector in both simulations and experiments. Because of the spin-coupling of the geometric phase, the incident left- and right-circular polarizations are deflected at symmetrical angles. When a voltage is applied across the graphene electrodes, the phase modulation disappears, and the $\mathrm{THz}$ wave is directly transmitted, realizing the switch of the deflection.

\section{Principle and Design}

The structure of the beam deflector (Figure 1a) has upper and lower substrates, which are made of fused silica with a thickness of $800 \mu \mathrm{m}$; the inner surfaces of the upper and lower substrates are covered with graphene and a photo-alignment layer, respectively. The electrode is formed from a few layers of graphene, which has good conductivity and high $\mathrm{THz}$ transmittance. The alignment material is azobenzene-based sulphonic dye SD1 [36], which produces a vertical alignment effect when illuminated by polarized ultraviolet light. A room-temperature LC nematic mixture (NJU-LDn-4) was adopted because its average birefringence band is greater than 0.3 over the $0.5-2.0 \mathrm{THz}$ band [37]. Applying photoalignment technology, an LC forms a periodic gradually distributed orientation thereby producing a blazed grating phase for $\mathrm{THz}$ beam deflection.

(a)

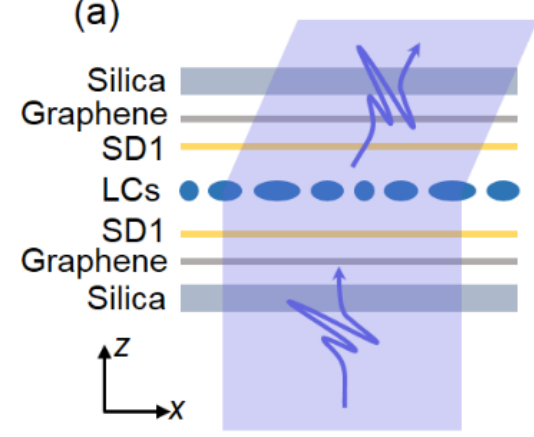

(c)

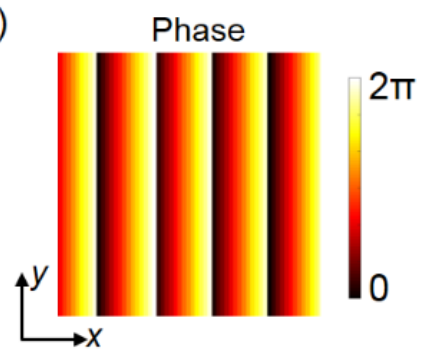

(b)

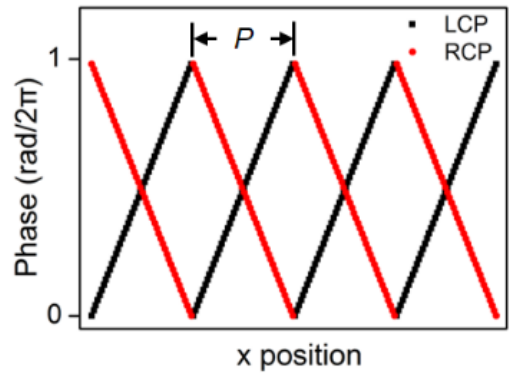

(d)

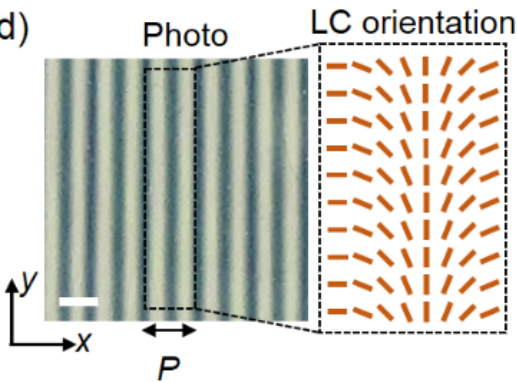

Figure 1. (a) Schematic of the beam deflector. (b) Required grating phase along the $x$-axis. (c) Phase distribution diagram of the designed grating. (d) Prepared LC sample under crossed polarizers and orientation distribution in the corresponding area.

The phase of the blazed grating comes from the geometric phase modulation formed by the orientation of LCs. A geometric phase, i.e., the Pancharatnam-Berry phase (PB phase) [38], is derived from photon spin-orbit coupling [39] and is achieved by controlling 
the orientation angle distribution of an anisotropic medium. For a uniformly oriented LC domain with an orientation angle of $\alpha$, its Jones matrix is [40]:

$$
\begin{aligned}
\mathbf{J} & =\mathbf{R}(-\alpha) \cdot\left(\begin{array}{cc}
\exp (-i \zeta) & 0 \\
0 & \exp (i \zeta)
\end{array}\right) \cdot \mathbf{R}(\alpha) \\
& =-i \sin \zeta \cdot\left(\begin{array}{cc}
\cos 2 \alpha & \sin 2 \alpha \\
\sin 2 \alpha & -\cos 2 \alpha
\end{array}\right)+\cos \zeta \cdot \mathbf{I}
\end{aligned}
$$

where $\mathbf{R}$ and I denote the rotation and identity matrices, respectively, and:

$$
\zeta=\pi \delta \mathrm{n} d / \lambda
$$

which is half the phase retardation obtained from the product of the LC birefringence $\delta n$, the cell thickness $d$, and the reciprocal of the operating wavelength $\lambda$.

The normalized Jones vectors of left- and right-handed circularly polarized (LCP and $\mathrm{RCP})$ waves, denoted as $\chi^{(+)}$and $\chi^{(-)}$, are:

$$
\chi^{( \pm)}=(1, \pm i)^{T} / \sqrt{2}
$$

When a circularly polarized $(\mathrm{CP})$ wave is incident, the outgoing wave is expressed as:

$$
\mathbf{E}_{\text {out }}=\mathbf{J} \cdot \chi^{( \pm)}=-i \sin \zeta \cdot \exp ( \pm i 2 \alpha) \cdot \chi^{( \pm)}+\cos \zeta \cdot \chi^{( \pm)}
$$

For an LCP incident wave, the outgoing wave divides into two parts: the first is the RCP wave with phase factor $\exp (i 2 \alpha)$, and the second is the LCP wave without phase modulation. The opposite is true for an RCP incident wave.

The required grating phase for beam deflection $\varphi$ is expressed as:

$$
\varphi=-2 \pi x \sin \theta / \lambda
$$

where $\theta$ and $x$ denote the deflection angle and the coordinate, respectively. After normalizing $\varphi$ by $2 \pi$, the phase distribution presents a periodic inclined phase (Figure $1 \mathrm{~b}$ ). We use the principle of geometric phase to realize the phase distribution. Because of the spin-conjugacy of the geometric phase, the grating phases for LCP and RCP incident waves are opposite (see Figure $1 b$ ). The period $P$ of the $2 \pi$ phase change determines the deflection angle $\theta$ for a given incident wavelength $\lambda$. We design a beam deflection phase with $\theta=13^{\circ}$ at $1 \mathrm{THz}$ (corresponding to $P=1333 \mu \mathrm{m}$ ). The normalized phase distribution (Figure 1c) features a periodic phase along the $x$-axis.

In preparing the device (Figure 2), a silica substrate is ultrasonically cleaned. Next, we transfer the few layers of graphene from a copper foil to the substrate. SD1 is spin-coated onto the graphene substrate. In forming the LC cell, a 500- $\mu \mathrm{m}$-thick Mylar film is used as the spacer. At $1 \mathrm{THz}$, the thickness satisfies the half-wave condition. Next, we use a digital micro-mirror device (DMD)-based microprojection exposure system to perform a multi-step overlapping polarized UV exposure [41]. The photo-alignment material SD1 is polarization-sensitive. When exposed to linearly polarized UV light, the SD1 molecules tend to be aligned perpendicular to the incident polarization. After the LC is infiltrated into the cell, SD1 further guides the spatial distribution of the LC to obtain the designed geometric phase distribution. Finally, we fill the cell with LC NJU-LDn-4 to form the beam deflector. The cross-polarized micrograph of the prepared sample and the LC orientation distribution in the corresponding area (Figure 1d) shows a light-dark gradient area that is consistent with the theoretical phase distribution. 


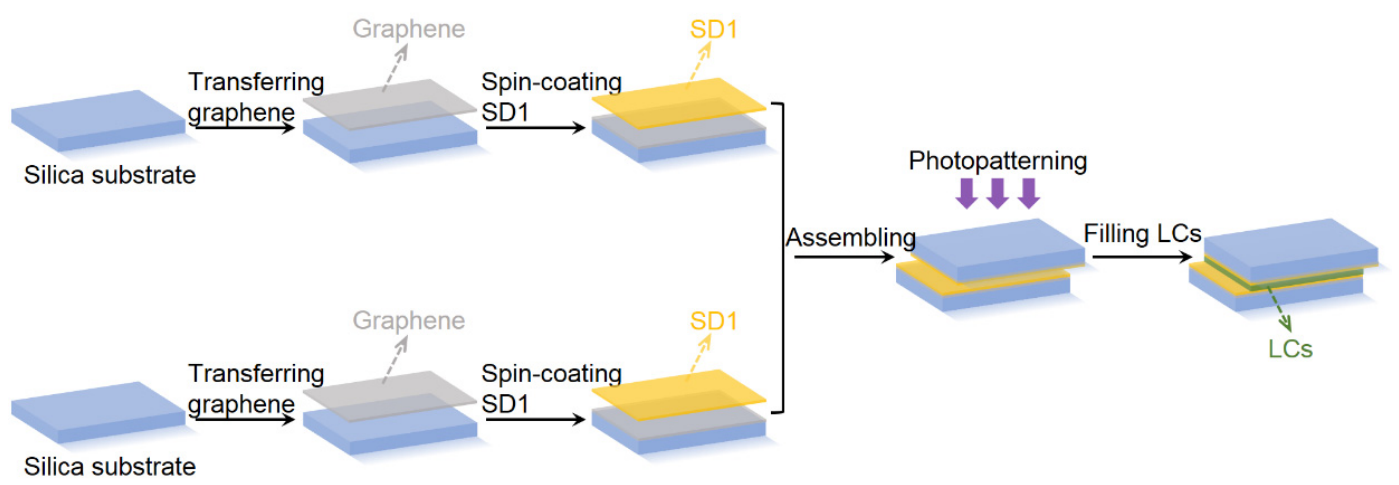

Figure 2. Schematic of the sample preparation process.

To verify the design, commercial electromagnetic simulations software (Lumerical FDTD Solutions) was used to simulate the lens numerically. The configuration is based on the LC orientation (Figure 1c) with a one-dimensional grating model profiled along the $x$-axis. Each LC pixel is considered as a rectangular shape of $50 \times 50 \times 500 \mu^{3}$. The LC material is an anisotropic dielectric material, where $n_{\mathrm{o}}=1.50+i 0.006, n_{\mathrm{e}}=1.81+i 0.001$, which is set by the LC orientation module in the software. The conductivity depends on the number of layers of the graphene, which affects the transmittance of the $\mathrm{THz}$ wave. The more layers there are, the higher the conductivity and the lower the transmittance are. Therefore, we use the graphene with 2-3 layers, which can not only ensure the conductive effect of the electrode but also allow more THZ waves to pass through, so as to maintain high performance of the device.

The Raman test has been mentioned in many previous articles on graphene as a transparent THZ electrode [32,35]. In our experiments, we used graphene materials with few layers provided by a mature company. The number of layers is determined and its performance can be guaranteed by measuring resistance and $\mathrm{THz}$ transmittance.

The plane wave is incident along the $z$-axis. The far-field intensity distributions under LCP and RCP incidence (Figure 3a,b, respectively) show clearly that the deflection angles are $-13^{\circ}$ and $+13^{\circ}$, which are consistent with the expected results. From diffraction effects, the intensity of the beam attenuates slightly with propagation.

To detect the beam deflection, we employed a scanning near-field $\mathrm{THz}$ microscope in which the $\mathrm{THz}$ waves are generated and detected using the photo-conductive antenna and microprobe, respectively. More specifically, a femtosecond pulse with a center wavelength of $780 \mathrm{~nm}$, pulse width of $90 \mathrm{fs}$, and pulse repetition frequency of $90 \mathrm{MHz}$, is divided into two beams; one is used as the pump beam for $\mathrm{THz}$ wave generation, and the other is used as the probe beam for $\mathrm{THz}$ wave detection. The transmitted $\mathrm{THz}$ wave covers a frequency band from 0.2 to $1.5 \mathrm{THz}$. After focusing and collimation by a parabolic mirror, the linearly polarized wave is converted into a $\mathrm{CP}$ wave by a quarter waveplate and subsequently detected by the microprobe. The scanning probe is used to obtain the $E_{\mathrm{x}}$ component distribution in the $x y$-plane. The sample is fixed on a $z$-axis stage and scans the electric field strength in the $x z$-plane. The results (Figure $3 c, d$ ) show the THz intensity distribution in the $x z$-plane displaying spin-coupled deflection. In the $x y$-plane, the Gaussian spot has a nonuniform intensity distribution that arises from the qualified LC alignment.

Furthermore, because the geometric phase is independent of the polarization, a dispersive deflection in a broadband is obtained. We measured the beam deflection of the device from 0.8 to $1.2 \mathrm{THz}$ (Figure 4a,b). A good spin-coupled beam deflection is achieved having different beam deflection angles at different frequencies. We plotted the measured deflection angle with frequency (Figure 4c). Under LCP and RCP incidences, the deflection angles gradually decrease as frequency increases, consistent with theory (black line). With the half-wave condition being attained at $1 \mathrm{THz}$, the device has its largest deflection conversion efficiency at $1 \mathrm{THz}$, reaching 94\% under LCP incidence (Figure 4d). At other frequencies, 
the efficiency gradually decreases with the gradual deviation from the half-wave condition. The measured efficiency is slightly smaller than the theoretical value (Figure 4d), which may be because the LC orientation is not perfect when under the influence of graphene.

(a)

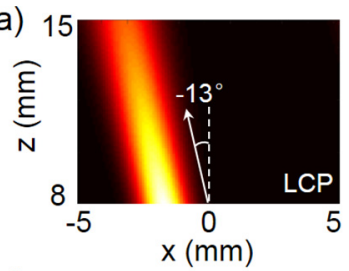

(c)

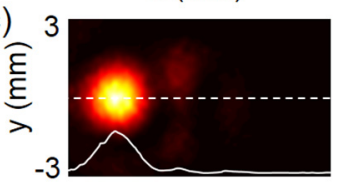

(e)

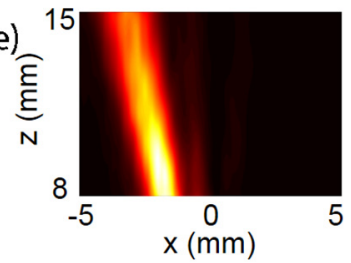

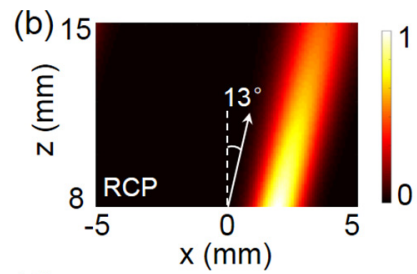
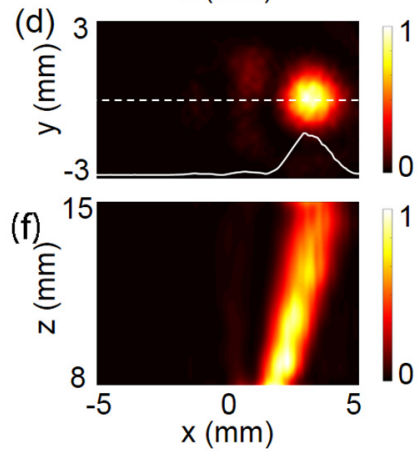

Figure 3. THz far-field intensity distributions: (a,b) Simulation results in the $x z$-plane under (a) LCP and (b) RCP incidence. (c,d) Measured results in the $x z$-plane and $x y$-plane $(z=15 \mathrm{~mm})$ under (c) LCP and (d) RCP incidence, (e,f) Simulation results in the xz-plane under (e) LCP and (f) RCP incidence. The white solid curves show the horizontal intensity distribution along the white dashed lines.

(a)
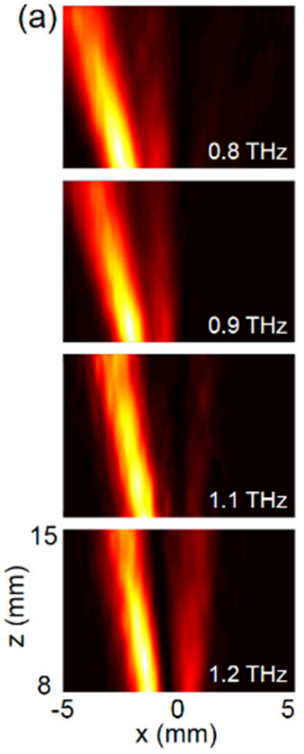

(b)
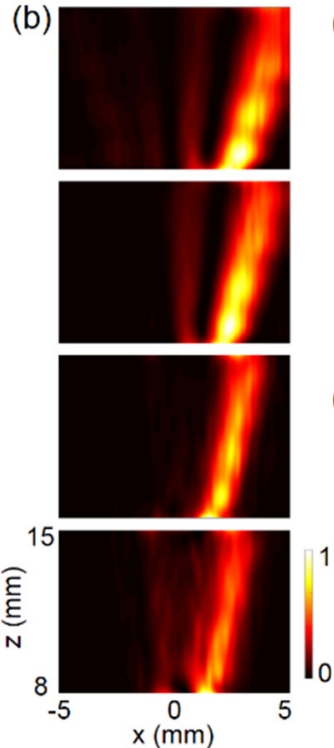

(c)

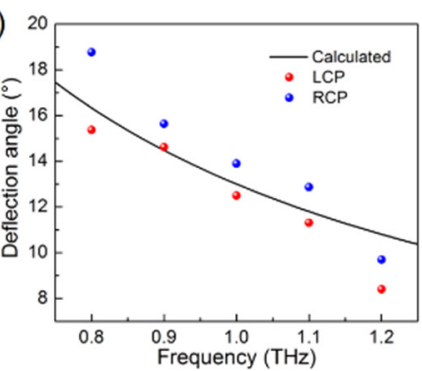

(d)

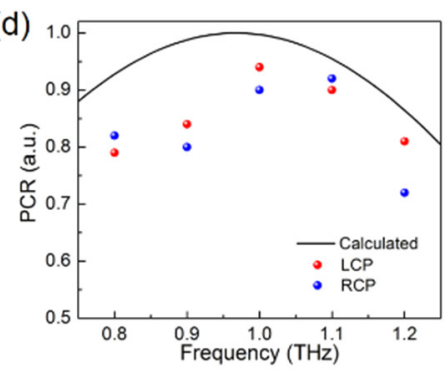

Figure 4. $(\mathbf{a}, \mathbf{b})$ Measured $\mathrm{THz}$ far-field intensity distribution in the $x z$-plane in the frequency range of 0.8-1.2 THz band under (a) LCP and (b) RCP incidence. (c,d) Dependence of (c) deflection angle and (d) polarization conversion efficiency with frequency.

The dependence relationship of the parameters such as efficiency, frequency and thickness of liquid crystal has been described by:

$$
\eta=\sin 2 \zeta=\sin 2(\pi \delta \text { ndf } / \mathrm{c}),
$$


where $\eta$ is the polarization conversion efficiency, $\delta n$ is the birefringence of the liquid crystal material, $\mathrm{d}$ is the thickness of liquid crystal, $\mathrm{c}$ is the speed of light, and $\mathrm{f}$ is the frequency.

Based on this equation, the polarization conversion efficiency at different liquid crystal thicknesses is provided in Figure 5. As shown in Figure 5, the polarization conversion efficiency reaches the maximum of $94 \%$ at $1 \mathrm{THz}$. Moreover, in our experiment, the signalto-noise ratio of the experimental setup is large at $1 \mathrm{THz}$. Therefore, we chose a crystal liquid layer thickness of $500 \mu \mathrm{m}$.

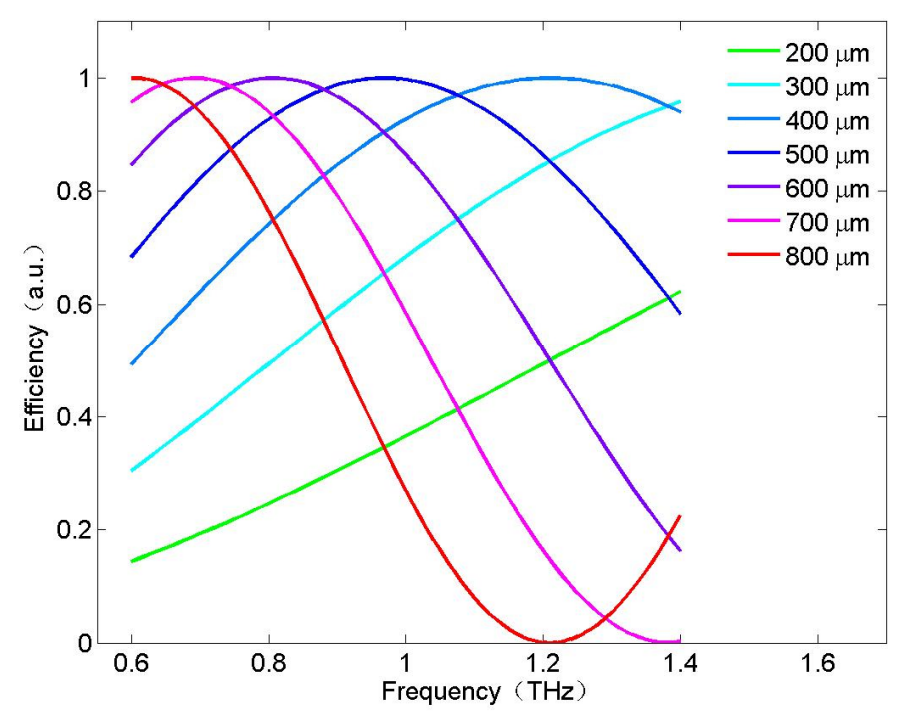

Figure 5. The polarization conversion efficiency at different liquid crystal thicknesses.

With the graphene electrode present, the beam deflector may be driven by an external voltage. We applied a square wave $(1 \mathrm{kHz})$ alternating signal during the experiment. Under a saturation voltage of $120 \mathrm{~V}$, the orientation of the LC is completely distributed along the $z$-axis (Figure 6a). In this instance, the beam deflection function disappears, and the transmitted $\mathrm{THz}$ wave is not modulated. The far-field intensity distributions in the $x y$-plane under LCP and RCP incidences (Figure $6 \mathrm{~b}, \mathrm{c}$, respectively) feature Gaussian intensity distributions, demonstrating dynamic switching. Because of the non-uniformity of the electric field, the $\mathrm{THz}$ wave is scattered by LCs and is not very uniform (Figure 6b,c).

(a)

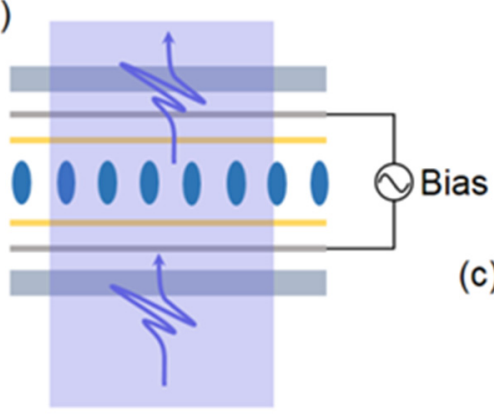

(b)

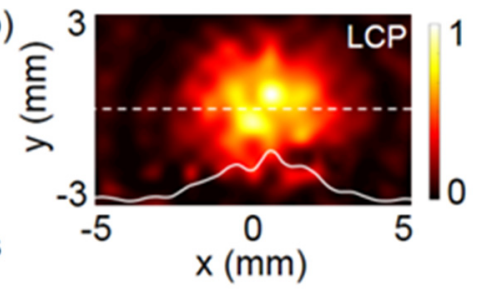

c)

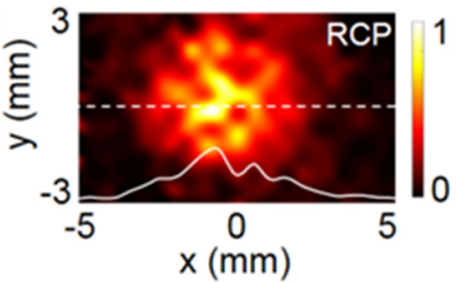

Figure 6. Results obtained after a saturation voltage is applied: (a) switchable function of the device; $(\mathbf{b}, \mathbf{c})$ far-field intensity distributions in the $x y$-plane under (b) LCP and (c) RCP incidence. The white solid line shows the horizontal intensity distribution along the white dashed line. 


\section{Results and Discussion}

The beam deflector is realized by the geometric phase of LCs. The phase has a double relationship with the orientation angle of the LC. Therefore, there is no need for complex simulations to determine the structural parameters. The alignment and fabrication of the LC devices follow well-established techniques, and hence, our device is very cheap and suitable for large-scale production; the modulation efficiency of the device is relatively high over a wide operating band. Further improvements are possible by optimizing the birefringence and absorptivity of the LC material. The device performs dynamic beam deflection, which will be of great benefit in $\mathrm{THz}$ communications and imaging.

\section{Conclusions}

In summary, we have realized a $\mathrm{THz}$ beam deflection device that exhibits a broad operating band and high efficiency. The conversion efficiency of dielectric metasurface devices can reach less than $80 \%$. Moreover, because the liquid crystal is non-resonant, the polarization conversion efficiency can reach more than $95 \%$ after optimizing the thickness of liquid crystal layer and using low loss liquid crystal materials, and has the advantage of simple processing compared with the metasurface. By exploiting the geometric phase distribution of the LCs, we have achieved a dispersive focusing effect in the $0.8-1.2 \mathrm{THz}$ band. Furthermore, by adding graphene electrodes on the upper and lower substrates with an external bias, we realized dynamic switching of the beam deflection. The device can be manufactured by a mature LC photo-aligning process and gainfully applied in the field of $\mathrm{THz}$ communication and imaging systems.

Author Contributions: Conceptualization, Y.S. and Y.W.; methodology, Y.S. and Y.W.; software, J.W. and Q.W.; validation, Y.S., X.Q. and J.W.; data curation, Y.W. and D.X.; writing-original draft preparation, Y.S. and Q.W.; writing - review and editing, Y.W. and D.X.; visualization, Y.S.; supervision, J.W. and D.X.; funding acquisition, Y.S. All authors have read and agreed to the published version of the manuscript.

Funding: The authors gratefully acknowledge the support of the Guangzhou Science and Technology Project (Grant 202102080473).

Data Availability Statement: The data presented in this study are contained within this article.

Acknowledgments: We gratefully acknowledge the support of $\mathrm{THz}$ measurements from Liquid Crystal and Nanophotonics Group of Nanjing University.

Conflicts of Interest: The authors declare no conflict of interest.

\section{References}

1. Federici, J.F.; Schulkin, B.; Huang, F.; Gary, D.; Barat, R.; Oliveira, F.; Zimdars, D. THz imaging and sensing for security applications-Explosives, weapons and drugs. Semicond. Sci. Technol. 2005, 20, S266. [CrossRef]

2. Kawase, K.; Ogawa, Y.; Watanabe, Y.; Inoue, H. Non-destructive terahertz imaging of illicit drugs using spectral fingerprints. Opt. Express 2003, 11, 2549-2554. [CrossRef] [PubMed]

3. Nagatsuma, T.; Ducournau, G.; Renaud, C.C. Advances in terahertz communications accelerated by photonics. Nat. Photonics 2016, 10, 371. [CrossRef]

4. Xie, J.Y.; Ye, W.C.; Zhou, L.J.; Guo, X.G.; Zang, X.F.; Chen, L.; Zhu, Y.M. A Review on Terahertz Technologies Accelerated by Silicon Photonics. Nanomaterials 2021, 11, 1646. [CrossRef]

5. Federici, J.; Moeller, L. Review of terahertz and subterahertz wireless communications. J. Appl. Phys. 2010, 107, 111101. [CrossRef]

6. D'Arco, A.; di Fabrizio, M.; Dolci, V.; Petrarca, M.; Lupi, S. THz Pulsed Imaging in Biomedical Applications. Condens. Matter 2020, 5, 25. [CrossRef]

7. Kim, Y.; Won, K.; An, J.; Hong, J.Y.; Kim, Y.; Choi, C.S.; Song, H.; Song, B.; Kim, H.S.; Bae, K.D.; et al. Large-area liquid crystal beam deflector with wide steering angle. Appl. Opt. 2020, 59, 7462-7468. [CrossRef]

8. Kleine-Ostmann, T.; Nagatsuma, T. A review on terahertz communications research. J. Infrared Millim. Terahertz Waves 2011, 32, 143-171. [CrossRef]

9. Koenig, S.; Lopez-Diaz, D.; Antes, J.; Boes, F.; Henneberger, R.; Leuther, A.; Tessmann, A.; Schmogrow, R.; Hillerkuss, D.; Palmer, R.; et al. Wireless sub-THz communication system with high data rate. Nat. Photonics 2013, 7, 977. [CrossRef]

10. Aieta, F.; Kats, M.A.; Genevet, P.; Capasso, F. Multiwavelength achromatic metasurfaces by dispersive phase compensation. Science 2015, 347, 1342-1345. [CrossRef] 
11. Zhang, H.; Zhang, X.; Xu, Q.; Tian, C.; Wang, Q.; Xu, Y.; Li, Y.; Gu, J.; Tian, Z.; Ouyang, C.; et al. High-Efficiency Dielectric Metasurfaces for Polarization-Dependent Terahertz Wavefront Manipulation. Adv. Opt. Mater. 2018, 6, 1700773. [CrossRef]

12. Zhang, H.; Zhang, X.; Xu, Q.; Wang, Q.; Xu, Y.; Wei, M.; Li, Y.; Gu, J.; Tian, Z.; Ouyang, C. Polarization-independent all-silicon dielectric metasurfaces in the terahertz regime. Photonics Res. 2018, 6, 24-29. [CrossRef]

13. Shrekenhamer, D.; Montoya, J.; Krishna, S.; Padilla, W. Four-Color Metamaterial Absorber THz Spatial Light Modulator. Adv. Opt. Mater. 2013, 1, 905-909. [CrossRef]

14. Chen, H.T.; Yang, H.; Singh, R.; Hara, J.F.; Azud, A.K.; Trugman, S.A.; Jia, Q.X.; Taylor, A.J. Tuning the Resonance in HighTemperature Superconducting Terahertz Metamaterials. Phys. Rev. Lett. 2010, 105, 247402. [CrossRef]

15. He, J.W.; Xie, Z.W.; Sun, W.F.; Wang, X.K.; Ji, Y.D.; Wang, S.; Lin, Y.; Zhang, Y. Terahertz Tunable Metasurface Lens Based on Vanadium Dioxide Phase Transition. Plasmonics 2016, 11, 1285-1290. [CrossRef]

16. Fu, Y.H.; Liu, A.Q.; Zhu, W.M.; Zhang, X.M.; Tsai, D.P.; Zhang, J.B.; Mei, T.; Kwong, D.L. A Micromachined Reconfi gurable Metamaterial via Reconfi guration of Asymmetric Split-Ring Resonators. Adv. Funct. Mater. 2011, 21, 3589. [CrossRef]

17. Monticone, F.; Estakhri, N.M.; Alu, A. Full Control of Nanoscale Optical Transmission with a Composite Metascreen. Phys. Rev. Lett. 2013, 110, 203903. [CrossRef]

18. Liu, S.; Cheng, Q.; Xu, Q.; Wang, T.Q.; Du, L.L.; Xu, K.L.Y.H.; Bao, D.; Fu, X.J.; Han, J.G.; Zhang, W.L.; et al. Free-Standing Metasurfaces for High-Effi ciency Transmitarrays for Controlling Terahertz Waves. Adv. Opt. Mater. 2016, 4, 384. [CrossRef]

19. Liu, S.; Noor, A.; Du, L.L.; Zhang, L.; Xu, Q.; Wang, K.L.T.Q.; Tian, Z.; Tang, W.X.; Han, J.G.; Zhang, W.L.; et al. Anomalous Refraction and Nondiffractive Bessel-Beam Generation of Terahertz Waves through Transmission-Type Coding Metasurfaces. ACS Photonics 2016, 3, 1968. [CrossRef]

20. Jia, M.; Wang, Z.; Li, H.T.; Wang, X.K.; Luo, W.J.; Sun, S.L.; Zhang, Y.; He, Q.; Zhou, L. Efficient manipulations of circularly polarized terahertz waves with transmissive metasurfaces. Light Sci. Appl. 2019, 8, 16. [CrossRef]

21. Jahani, S.; Jacob, Z. All-dielectric metamaterials. Nat. Nanotech. 2016, 11, 23. [CrossRef]

22. Headland, D.; Carrasco, E.; Nirantar, S.; Withayachumnankul, W.; Gutruf, P.; Schwarz, J.; Abbott, D.; Bhaskaran, M.; Sriram, S.; Perruisseau, J.; et al. Dielectric Resonator Reflectarray as High-Efficiency Nonuniform Terahertz Metasurface. ACS Photonics 2016, 3, 1019. [CrossRef]

23. Scheffer, T.; Nehring, J. A new, highly multiplexable liquid crystal display. Appl. Phys. Lett. 1984, 45, 1021-1023. [CrossRef]

24. Rao, L.; Ge, Z.; Wu, S.-T.; Lee, S.H. Low voltage blue-phase liquid crystal displays. Appl. Phys. Lett. 2009, 95, 231101. [CrossRef]

25. Zhang, Z.; You, Z.; Chu, D. Fundamentals of phase-only liquid crystal on silicon (LCOS) devices. Light Sci. Appl. 2014, 3, e213. [CrossRef]

26. Gu, D.; Wen, B.; Mahajan, M.; Taber, D.; Winker, B.; Guthals, D.; Campbell, B.; Sox, D. High power liquid crystal spatial light modulators. In Proceedings of the Advanced Wavefront Control: Methods, Devices, and Applications IV, San Diego, CA, USA, 14-15 August 2006; International Society for Optics and Photonics: Bellingham, WA, USA, 2006; p. 630602.

27. Chen, C.Y.; Pan, C.L.; Hsieh, C.F.; Lin, Y.F.; Pan, R.P. Liquid-crystal-based terahertz tunable Lyot filter. Appl. Phys. Lett. 2006, 88, 101107. [CrossRef]

28. Chen, C.-Y.; Hsieh, C.-F.; Lin, Y.-F.; Pan, R.-P.; Pan, C.-L. Magnetically tunable room-temperature $2 \pi$ liquid crystal terahertz phase shifter. Opt. Express 2004, 12, 2625-2630. [CrossRef] [PubMed]

29. Lin, X.-w.; Wu, J.-b.; Hu, W.; Zheng, Z.-g.; Wu, Z.-j.; Zhu, G.; Xu, F.; Jin, B.-b.; Lu, Y.-Q. Self-polarizing terahertz liquid crystal phase shifter. AIP Adv. 2011, 1, 032133. [CrossRef]

30. Yang, C.-S.; Tang, T.-T.; Pan, R.-P.; Yu, P.; Pan, C.-L. Liquid crystal terahertz phase shifters with functional indium-tin-oxide nanostructures for biasing and alignment. Appl. Phys. Lett. 2014, 104, 141106. [CrossRef]

31. Du, Y.; Tian, H.; Cui, X.; Wang, H.; Zhou, Z.-X. Electrically tunable liquid crystal terahertz phase shifter driven by transparent polymer electrodes. J. Mater. Chem. C 2016, 4, 4138-4142. [CrossRef]

32. Wu, Y.; Ruan, X.; Chen, C.-H.; Shin, Y.J.; Lee, Y.; Niu, J.; Liu, J.; Chen, Y.; Yang, K.-L.; Zhang, X.; et al. Graphene/liquid crystal based terahertz phase shifters. Opt. Express 2013, 21, 21395-21402. [CrossRef]

33. Ge, S.J.; Chen, P.; Shen, Z.X.; Sun, W.F.; Wang, X.K.; Hu, W.; Zhang, Y.; Lu, Y.Q. Terahertz vortex beam generator based on a photopatterned large birefringence liquid crystal. Opt. Express 2017, 25, 12349-12356. [CrossRef]

34. Shen, Z.; Zhou, S.; Ge, S.; Duan, W.; Ma, L.; Lu, Y.; Hu, W. Liquid crystal tunable terahertz lens with spin-selected focusing property. Opt. Express 2019, 27, 8800-8807. [CrossRef]

35. Wang, L.; Lin, X.-W.; Hu, W.; Shao, G.-H.; Chen, P.; Liang, L.-J.; Jin, B.-B.; Wu, P.-H.; Qian, H.; Lu, Y.-N.; et al. Broadband tunable liquid crystal terahertz waveplates driven with porous graphene electrodes. Light Sci. Appl. 2015, 4, e253. [CrossRef]

36. Shen, Z.-X.; Tang, M.-J.; Chen, P.; Zhou, S.-H.; Ge, S.-J.; Duan, W.; Wei, T.; Liang, X.; Hu, W.; Lu, Y.-Q. Planar Terahertz Photonics Mediated by Liquid Crystal Polymers. Adv. Opt. Mater. 2020, 8, 1902124. [CrossRef]

37. Wang, L.; Lin, X.-W.; Liang, X.; Wu, J.-B.; Hu, W.; Zheng, Z.-g.; Jin, B.-b.; Qin, Y.-q.; Lu, Y.-q. Large birefringence liquid crystal material in terahertz range. Opt. Mater. Express 2012, 2, 1314-1319. [CrossRef]

38. Berry, M.V. The Adiabatic Phase and Pancharatnam's Phase for Polarized Light. J. Mod. Opt. 1987, 34, 1401-1407. [CrossRef]

39. Bliokh, K.Y.; Rodríguez-Fortuño, F.J.; Nori, F.; Zayats, A.V. Spin-orbit interactions of light. Nat. Photonics 2015, 9, 796. [CrossRef]

40. Yeh, P.; Gu, C. Optics of Liquid Crystal Displays; John Wiley \& Sons: Hoboken, NJ, USA, 2009; Volume 67.

41. Ji, W.; Lee, C.-H.; Chen, P.; Hu, W.; Ming, Y.; Zhang, L.; Lin, T.-H.; Chigrinov, V.; Lu, Y.-Q. Meta-q-plate for complex beam shaping. Sci. Rep. 2016, 6, 25528. [CrossRef] 